\title{
Examining Customer Referral Reward Programs: Does Reward Fairness Matter?
}

\author{
David Dose and Gianfranco Walsh
}

\begin{abstract}
Customer referral reward programs (CRPs) are an important and widespread marketing tool in various service industries. However, the effectiveness of CRPs is contingent on the success of the recommender-receiver interaction. This research focuses on reward schemes, that is, systems that involve payments from service providers to customers that help the provider acquire new customers. Specifically, the authors investigate situations in which the referral reward is split between recommender and referral receiver, whereby both parties negotiate how much of the referral reward each party receives. In studying referral-reward allocations the present research investigates an important issue regarding the effectiveness of CRPs in general and the recommender-receiver interaction in particular. Building on equity aversion models, the authors show that rewarding only the recommender brings about perceptions of unfairness on part of the referral receiver, resulting in disruptive behavior and inefficient referral processes. The results expand the existing theoretical assumption that CRPs are continuously controlled by firms and indicate that due to biases in fairness perceptions the interaction between recommender and referral receiver remains unpredictable and difficult to control for referring firms. However, results also reveal that tie strength cushions the effect of biased fairness perceptions on successful referral processes.
\end{abstract}

D. Dose $(\bowtie) \cdot$ G. Walsh

University of Jena, Jena, Germany

e-mail: david.dose@uni-jena.de; walsh@uni-jena.de 